\title{
SPATIAL REGULARITY OF SEMIGROUPS GENERATED BY LÉVY TYPE OPERATORS
}

\author{
MINGJIE LIANG JIAN WANG
}

\begin{abstract}
We apply the probabilistic coupling approach to establish the spatial regularity of semigroups associated with Lévy type operators, by assuming that the martingale problem of Lévy type operators is well posed. In particular, we can prove the Lipschitz continuity of the semigroups under Hölder continuity of coefficients, even when the Lévy kernel corresponding to Lévy type operators is singular.
\end{abstract}

Keywords: Lévy type operator; coupling; spatial regularity; martingale problem MSC 2010: 60J25; 60J75.

\section{Introduction and Main Results}

We consider the following Lévy type operator

$$
L f(x)=\int\left(f(x+z)-f(x)-\langle\nabla f(x), z\rangle \mathbb{1}_{B(0,1)}(z)\right) c(x, z) \nu(d z),
$$

where $\nu$ is a Lévy measure, i.e., $\nu(\{0\})=0$ and $\int\left(1 \wedge|z|^{2}\right) \nu(d z)<\infty$, and $(x, z) \mapsto$ $c(x, z)$ is a continuous function such that $c(x, z) \in\left(c_{*}, c^{*}\right)$ for some constants $0<$ $c_{*} \leq c^{*}<\infty$. The operator $L$ is a non-local version of the classic second order elliptic operator with non-divergence form, and it has been attracted a lot of attentions in the community of analysis and PDEs, see, e.g. [11, 12]. In the probability theory, $L$ is corresponding to an infinitesimal generator of a large class of Feller processes, see the monographs $[9,23]$. The operator $L$ is also connected with the following pure jump process on $\mathbb{R}^{d}$ :

$$
\begin{aligned}
d J_{t}= & \int_{0}^{\infty} \int_{\{|z| \leq 1\}} z \mathbb{1}_{\left[0, c\left(J_{t-}, z\right)\right]}(r) \widetilde{N}(d z, d r, d t) \\
& +\int_{0}^{\infty} \int_{\{|z|>1\}} z \mathbb{1}_{\left[0, c\left(J_{t-}, z\right)\right]}(r) N(d z, d r, d t), \quad t \geq 0,
\end{aligned}
$$

where $N(d z, d r, d t)$ is a Poisson random measure on $\mathbb{R}^{d} \times[0, \infty) \times[0, \infty)$ with intensity measure $\nu(d z) d r d t$, and $\widetilde{N}(d z, d r, d t)=N(d z, d r, d t)-\nu(d z) d r d t$ is the compensated Poisson random measure. See [17, 18, 34, 35] for more details. The pure jump process of the form as $\left(J_{t}\right)_{t \geq 0}$ has been found very useful in applications; for instance, $\left(J_{t}\right)_{t \geq 0}$ plays a crucial role as the control when proving Freidlin-Wentzell type large deviation for stochastic differential equations with jumps via weak convergence approach, see e.g. [10].

M. Liang: College of Mathematics and Informatics, Fujian Normal University, 350007 Fuzhou, P.R. China. liangmingjie@aliyun.com.

J. Wang: College of Mathematics and Informatics \& Fujian Key Laboratory of Mathematical Analysis and Applications (FJKLMAA), Fujian Normal University, 350007 Fuzhou, P.R. China. jianwang@f jnu.edu.cn. 
The aim of this paper is to establish the spatial regularity of semigroups associated with the operator $L$ given by (1.1). We will adopt the probabilistic coupling approach, which recently has been extensively studied in [20, 21, 22, 33]. To study analytic properties for Lévy type operators via probabilistic method, as one of the standing assumptions, the existence of a strong Markov process associated with Lévy type operators was assumed. For example, see [5, 6, 7, 21, 33], where the strong Markov property played an important role. Similarly, throughout this paper we shall assume that the martingale problem for $\left(L, C_{b}^{2}\left(\mathbb{R}^{d}\right)\right)$ is well posed (see Section 2.2 below for its definition). In particular, there is a strong Markov process $X:=\left(\left(X_{t}\right)_{t \geq 0},\left(\mathbb{P}^{x}\right)_{x \in \mathbb{R}^{d}}\right)$, whose generator is just the Lévy type operator $L$. Below, for any $f \in B_{b}\left(\mathbb{R}^{d}\right)$ (the set of bounded measurable functions on $\mathbb{R}^{d}$ ), let

$$
P_{t} f(x)=\mathbb{E}^{x} f\left(X_{t}\right), \quad x \in \mathbb{R}^{d}, t \geq 0
$$

be the semigroups corresponding to the operator $L$.

To state the contribution of our paper, we consider the following Lévy measure partly motivated by [14]. (Actually, the paper [14] treated Lévy kernel case but with a fixed order, see $[14,(1.2)]$.) Suppose that there are constants $c_{1}, c_{2}>0$ and $0<\alpha_{1} \leq \alpha_{2}<2$ such that

$$
\frac{c_{1}}{|z|^{d+\alpha_{1}}} \mathbb{1}_{V_{\xi}}(z) d z \leq \nu(d z) \leq \frac{c_{2}}{|z|^{d+\alpha_{2}}} d z
$$

where

$$
V_{\xi}=\left\{z \in \mathbb{R}^{d}:|z| \leq 1 \text { and }\langle z, \xi\rangle \geq \delta|z|\right\}
$$

with $\xi \in \mathbb{S}^{d-1}$ and constant $\delta \in(0,1)$.

Theorem 1.1. Under the assumption (1.3), we define for any $r>0$,

$$
w(r)= \begin{cases}\sup _{x, y \in \mathbb{R}^{d:|x-y|=r}} \int_{\{|z| \leq 1\}}|z|^{2}|c(x, z)-c(y, z)| \nu(d z), & \alpha_{2} \in[1,2) ; \\ \sup _{x, y \in \mathbb{R}^{d}:|x-y|=r} \int_{\{|z| \leq 1\}}|z||c(x, z)-c(y, z)| \nu(d z), & \alpha_{2} \in(0,1) .\end{cases}
$$

Then the following statements hold.

(1) If $\alpha_{1} \in(1,2)$ and

$$
\lim _{r \rightarrow 0} w(r) r^{\alpha_{1}-2} \log ^{1+\theta}(1 / r)=0
$$

for some $\theta>0$, then there exists a constant $C>0$ such that for all $f \in$ $B_{b}\left(\mathbb{R}^{d}\right)$ and $t>0$,

$$
\sup _{x \neq y} \frac{\left|P_{t} f(x)-P_{t} f(y)\right|}{|x-y|} \leq C\|f\|_{\infty}(t \wedge 1)^{-1 / \alpha_{1}}\left[\log ^{(1+\theta) / \alpha_{1}}\left(\frac{1}{t \wedge e}\right)\right] .
$$

(2) If $\alpha_{1} \in[1,2)$ and

$$
\lim _{r \rightarrow 0} w(r) r^{\alpha_{1}-2} \log (1 / r)=0,
$$

then, for any $\theta>\mathbb{1}_{\left\{\alpha_{1}=1\right\}}$, there exists a constant $C>0$ such that for all $f \in B_{b}\left(\mathbb{R}^{d}\right)$ and $t>0$,

$$
\sup _{x \neq y} \frac{\left|P_{t} f(x)-P_{t} f(y)\right|}{|x-y||\log | x-y||^{\theta}} \leq C\|f\|_{\infty}(t \wedge 1)^{-1 / \alpha_{1}}\left[\log ^{-\theta+\left(1 / \alpha_{1}\right)}\left(\frac{1}{t \wedge e}\right)\right] \text {. }
$$


(3) If $\alpha_{2} \in(0,1)$ and

$$
\lim _{r \rightarrow 0} w(r) r^{\alpha_{1}-1}=0
$$

then for any $\theta \in\left(0, \alpha_{1}\right)$, there exists a constant $C>0$ such that for all $f \in B_{b}\left(\mathbb{R}^{d}\right)$ and $t>0$,

$$
\sup _{x \neq y} \frac{\left|P_{t} f(x)-P_{t} f(y)\right|}{|x-y|^{\theta}} \leq C\|f\|_{\infty}(t \wedge 1)^{-\theta / \alpha_{1}} .
$$

Note that, the continuity assumptions on $w(r)$ in the theorem above are weaker than those on the function $x \mapsto c(x, z)$ (uniformly with respect to $z$ ). The latter was used to study the Hölder continuity of solutions to a class of second order non-linear elliptic integro-differential equations in [3, Theorem 3.1]. See also [2, Section 4.2] for more details. We also mention that similar assumptions (but a little stronger) on $w(r)$ have been adopted to study the pathwise uniqueness of solution to stochastic differential equations driven by the pure jump process $\left(J_{t}\right)_{t \geq 0}$ of the form $(1.2)$, see [17, Theorem 3.1], [34, Theorem 1.2] or [35, Theorem 2.1].

1.1. Applications. A Borel measurable function $u$ on $\mathbb{R}^{d}$ is called harmonic with respect to $L$, if $P_{t} u(x)=u(x)$ for all $x \in \mathbb{R}^{d}$ and $t>0$. The following result is a direct consequence of Theorem 1.1.

Corollary 1.2. Under the setting of Theorem 1.1, we have the following three statements.

(1) If assumptions in the first assertion of Theorem 1.1 hold, then any bounded measurable function $u$ is Lipschitz continuous.

(2) If assumptions in the second assertion of Theorem 1.1 hold, then, for any $\theta>\mathbb{1}_{\left\{\alpha_{1}=1\right\}}$, any bounded measurable function $u$ is $r \log ^{\theta}|r|$-order continuous.

(3) If assumptions in the third assertion of Theorem 1.1 hold, then for any $\varepsilon>0$, any bounded measurable function $u$ is $\left(\alpha_{1}-\varepsilon\right)$-Hölder continuous.

Next, we apply Theorem 1.1 to prove the following Liouville theorem. See [29, Theorem 2.1] for the related discussion for general symmetric $\alpha$-stable operators.

Corollary 1.3. Consider the setting of Theorem 1.1, and let $u$ be a harmonic function respect to the operator $L$. Suppose that there exists a constant $c>0$ such that for all $r \geq 1$,

$$
\|u\|_{L^{\infty}\left(B_{r}(0), d x\right)} \leq c r^{\beta}
$$

where $B_{r}(0)=\left\{z \in \mathbb{R}^{d}:|z|<r\right\}$ and $0 \leq \beta<\alpha_{2}$. If assumptions in any assertion of Theorem 1.1 hold, then $u$ is a polynomial of degree at most $\lfloor\beta\rfloor$, where $\lfloor x\rfloor$ denotes the integer part of $x$.

1.2. Perturbation result. To further illustrate the power of the coupling approach, we next give a perturbation result corresponding to Theorem 1.1. Below we will consider the following Lévy type operator

$$
L_{*} f(x)=\int\left(f(x+z)-f(x)-\langle\nabla f(x), z\rangle \mathbb{1}_{B(0,1)}(z)\right)(c(x, z) \nu(d z)+\mu(x, d z)),
$$

where $c(x, z)$ and $\nu(d z)$ are assumed same as those in the beginning of this section, and $\mu(x, d z)$ is a Lévy kernel on $\mathbb{R}^{d}$ satisfying that

$$
\sup _{x \in \mathbb{R}^{d}} \int\left(1 \wedge|z|^{2}\right) \mu(x, d z)<\infty
$$


and for any $h \in C_{b}^{2}\left(\mathbb{R}^{d}\right)$, the function

$$
x \mapsto \int_{\mathbb{R}^{d}} h(z) \frac{|z|^{2}}{1+|z|^{2}} \mu(x, d z)
$$

is continuous. We assume that the martingale problem for $\left(L_{*}, C_{b}^{2}\left(\mathbb{R}^{d}\right)\right)$ is wellposed. Clearly, the operator $L_{*}$ is just the operator $L$ perturbed by the Lévy kernel $\mu(x, d z)$. We emphasize that, we do not assume that the Lévy kernel $\mu(x, d z)$ is absolutely continuous with respect to the Lebesgue measure.

For any $r>0$, we define

$$
w_{\mu}(r)=\sup _{x, y \in \mathbb{R}^{d}:|x-y|=r} \int_{\{|z| \leq 1\}}|z|^{2}|\mu(x, d z)-\mu(y, d z)| ;
$$

if additionally

$$
\sup _{x \in \mathbb{R}^{d}} \int_{\{|z| \leq 1\}}|z| \mu(x, d z)<\infty,
$$

then $w_{\mu}(r)$ above is replaced by

$$
w_{\mu}(r)=\sup _{x, y \in \mathbb{R}^{d}:|x-y|=r} \int_{\{|z| \leq 1\}}|z||\mu(x, d z)-\mu(y, d z)| .
$$

Theorem 1.4. Under assumptions of Theorem 1.1 and notations above, define

$$
w_{*}(r)=w(r)+w_{\mu}(r), \quad r>0 .
$$

Then

(1) the first assertion of Theorem 1.1 holds, if $\alpha_{1} \in(1,2)$ and (1.5) holds with $w_{*}(r)$ replacing $w(r)$.

(2) the second assertion of Theorem 1.1 holds, if $\alpha_{1} \in[1,2)$ and (1.6) holds with $w_{*}(r)$ replacing $w(r)$.

(3) the third assertion of Theorem 1.1 holds, if $\alpha_{2} \in(0,1),(1.9)$ is satisfied, and (1.7) holds with $w_{*}(r)$ replacing $w(r)$.

The remainder of this paper is arranged as follows. The next section is devoted to the construction of a new coupling operator for the Lévy type operator $L$ given by (1.1), and the existence of coupling process on $\mathbb{R}^{2 d}$ associated with the constructed coupling operator. In Section 3, we first present some preliminary estimates for the coupling operator, and then give general results for the regularity of associated semigroups, by making full use of the coupling operator and the coupling process constructed in Section 2. Finally, the proofs of all results above are given in the last part of Section 3.

\section{Coupling operator And COUpling process for LÉvy type operators}

This section is split into two parts. We first present a new coupling operator for the Lévy type operator $L$ given by (1.1), and then prove the existence of coupling process on $\mathbb{R}^{2 d}$ associated with the constructed coupling operator. 
2.1. Coupling operator for Lévy type operator. The construction below is heavily based on the refined basic coupling for stochastic differential equations driven by additive Lévy noises first introduced in [22]. See [20] for the recent study on stochastic differential equations driven by multiplicative Lévy noises. However, the Lévy type operator $L$ given by (1.1) essentially is different from stochastic differential equations with jumps, and the main difficulty here is due to that the coefficient $c(x, z)$ in the operator $L$ depends on both space variables, which requires a new idea for the construction of a coupling operator.

For any $x, y, z, u \in \mathbb{R}^{d}$, define

$$
\begin{aligned}
c(x, y, u, z) & =c(x, z) \wedge c(y, z) \wedge c(x, z-u) \wedge c(y, z-u), \\
\nu_{u}(d z) & =\nu \wedge\left(\delta_{u} * \nu\right)(d z), \\
\mu_{x, y, u}(d z) & =c(x, y, u, z) \nu_{u}(d z), \\
\tilde{\nu}_{x, y}(d z) & =(c(x, z) \wedge c(y, z)) \nu(d z) .
\end{aligned}
$$

In particular, for any $x, y, z, u \in \mathbb{R}^{d}$,

$$
c(x, y, u, z)=c(x, y,-u, z-u) .
$$

The function $c(x, y, u, z)$ and the kernel $\mu_{x, y, u}(d z)$ are crucial in the construction of the coupling operator below.

For any $x, y \in \mathbb{R}^{d}$ and $\kappa>0$, let

$$
(x-y)_{\kappa}=\left(1 \wedge \frac{\kappa}{|x-y|}\right)(x-y) \text {. }
$$

We consider the jump system as follows

$$
(x, y) \longrightarrow \begin{cases}\left(x+z, y+z+(x-y)_{\kappa}\right), & \frac{1}{2} \mu_{x, y,(y-x)_{\kappa}}(d z) \\ \left(x+z, y+z+(y-x)_{\kappa}\right), & \frac{1}{2} \mu_{x, y,(x-y)_{\kappa}}(d z) \\ (x+z, y+z), & \left(\tilde{\nu}_{x, y}-\frac{1}{2} \mu_{x, y,(y-x)_{\kappa}}-\frac{1}{2} \mu_{x, y,(x-y)_{\kappa}}\right)(d z) \\ (x+z, y), & \tilde{c}(x, y, z) \nu(d z) ; \\ (x, y+z), & \tilde{c}(y, x, z) \nu(d z),\end{cases}
$$

where

$$
\tilde{c}(x, y, z)=c(x, z)-c(x, z) \wedge c(y, z) .
$$


Furthermore, for any $h \in C_{b}^{2}\left(\mathbb{R}^{2 d}\right)$ and $x, y \in \mathbb{R}^{d}$, we define the following operator associated with the jumping system above

$$
\begin{aligned}
& \widetilde{L} h(x, y) \\
& =\frac{1}{2} \int\left(h\left(x+z, y+z+(x-y)_{\kappa}\right)-h(x, y)-\left\langle\nabla_{x} h(x, y), z\right\rangle \mathbb{1}_{\{|z| \leq 1\}}\right. \\
& \left.-\left\langle\nabla_{y} h(x, y), z+(x-y)_{\kappa}\right\rangle \mathbb{1}_{\left\{\left|z+(x-y)_{\kappa}\right| \leq 1\right\}}\right) \mu_{x, y,(y-x)_{\kappa}}(d z) \\
& +\frac{1}{2} \int\left(h\left(x+z, y+z+(y-x)_{\kappa}\right)-h(x, y)-\left\langle\nabla_{x} h(x, y), z\right\rangle \mathbb{1}_{\{|z| \leq 1\}}\right. \\
& \left.-\left\langle\nabla_{y} h(x, y), z+(y-x)_{\kappa}\right\rangle \mathbb{1}_{\left\{\left|z+(y-x)_{\kappa}\right| \leq 1\right\}}\right) \mu_{x, y,(x-y)_{\kappa}}(d z) \\
& +\int\left(h(x+z, y+z)-h(x, y)-\left\langle\nabla_{x} h(x, y), z\right\rangle \mathbb{1}_{\{|z| \leq 1\}}\right. \\
& \left.-\left\langle\nabla_{y} h(x, y), z\right\rangle \mathbb{1}_{\{|z| \leq 1\}}\right)\left(\tilde{\nu}_{x, y}-\frac{1}{2} \mu_{x, y,(x-y)_{\kappa}}-\frac{1}{2} \mu_{x, y,(y-x)_{\kappa}}\right)(d z) \\
& +\int\left(h(x+z, y)-h(x, y)-\left\langle\nabla_{x} h(x, y), z\right\rangle \mathbb{1}_{\{|z| \leq 1\}}\right) \tilde{c}(x, y, z) \nu(d z) \\
& +\int\left(h(x, y+z)-h(x, y)-\left\langle\nabla_{y} h(x, y), z\right\rangle \mathbb{1}_{\{|z| \leq 1\}}\right) \tilde{c}(y, x, z) \nu(d z),
\end{aligned}
$$

where $\nabla_{x} h(x, y)$ and $\nabla_{y} h(x, y)$ are defined as the gradient of $h(x, y)$ with respect to $x \in \mathbb{R}^{d}$ and $y \in \mathbb{R}^{d}$ respectively.

We will claim that

Proposition 2.1. The operator $\widetilde{L}$ defined by (2.2) is indeed a coupling operator of $L$; that is, for any $f, g \in C_{b}^{2}\left(\mathbb{R}^{d}\right)$, letting $h(x, y)=f(x)+g(y)$ for all $x, y \in \mathbb{R}^{d}$, it holds that

$$
\widetilde{L} h(x, y)=L f(x)+L g(y) .
$$

Proof. The proof is similar to that in [22, Section 2.1] with slight modifications, and for the sake of completeness we present it here. Let $h(x, y)=g(y)$ for any $x, y \in \mathbb{R}^{d}$, where $g \in C_{b}^{2}\left(\mathbb{R}^{d}\right)$. Then, according to $(2.2)$,

$$
\begin{aligned}
\widetilde{L} h(x, y)=\frac{1}{2} \int\left(g\left(y+z+(x-y)_{\kappa}\right)-g(y)\right. \\
\left.\quad-\left\langle\nabla g(y), z+(x-y)_{\kappa}\right\rangle \mathbb{1}_{\left\{\left|z+(x-y)_{\kappa}\right| \leq 1\right\}}\right) \mu_{x, y,(y-x)_{\kappa}}(d z) \\
+\frac{1}{2} \int\left(g\left(y+z+(y-x)_{\kappa}\right)-g(y)\right. \\
\left.\quad-\left\langle\nabla g(y), z+(y-x)_{\kappa}\right\rangle \mathbb{1}_{\left\{\left|z+(y-x)_{\kappa}\right| \leq 1\right\}}\right) \mu_{x, y,(x-y)_{\kappa}}(d z) \\
+\int\left(g(y+z)-g(y)-\langle\nabla g(y), z\rangle \mathbb{1}_{\{|z| \leq 1\}}\right) \\
\quad \times\left(\tilde{\nu}_{x, y}-\frac{1}{2} \mu_{x, y,(x-y)_{\kappa}}-\frac{1}{2} \mu_{x, y,(y-x)_{\kappa}}\right)(d z) \\
+\int\left(g(y+z)-g(y)-\langle\nabla g(y), z\rangle \mathbb{1}_{\{|z| \leq 1\}}\right) \tilde{c}(y, x, z) \nu(d z) .
\end{aligned}
$$


Changing the variables $z+(x-y)_{\kappa} \rightarrow u$ and $z+(y-x)_{\kappa} \rightarrow u$ respectively and using Lemma 2.2 below in the first two terms of the right hand side of the equality above lead to

$$
\begin{aligned}
& \widetilde{L} h(x, y) \\
& =\frac{1}{2} \int\left(g(y+u)-g(y)-\langle\nabla g(y), u\rangle \mathbb{1}_{\{|u| \leq 1\}}\right) \\
& \times c\left(x, y,(y-x)_{\kappa}, u-(x-y)_{\kappa}\right) \nu_{(y-x)_{\kappa}}\left(d\left(u-(x-y)_{\kappa}\right)\right) \\
& +\frac{1}{2} \int\left(g(y+u)-g(y)-\langle\nabla g(y), u\rangle \mathbb{1}_{\{|u| \leq 1\}}\right) \\
& \times c\left(x, y,(x-y)_{\kappa}, u-(y-x)_{\kappa}\right) \nu_{(x-y)_{\kappa}}\left(d\left(u-(y-x)_{\kappa}\right)\right) \\
& +\int\left(g(y+z)-g(y)-\langle\nabla g(y), z\rangle \mathbb{1}_{\{|z| \leq 1\}}\right) \\
& \times\left(\tilde{\nu}_{x, y}-\frac{1}{2} \mu_{x, y,(x-y)_{\kappa}}-\frac{1}{2} \mu_{x, y,(y-x)_{\kappa}}\right)(d z) \\
& +\int\left(g(y+z)-g(y)-\langle\nabla g(y), z\rangle \mathbb{1}_{\{|z| \leq 1\}}\right) \tilde{c}(y, x, z) \nu(d z) \\
& =\frac{1}{2} \int\left(g(y+u)-g(y)-\langle\nabla g(y), u\rangle \mathbb{1}_{\{|u| \leq 1\}}\right) c\left(x, y,(x-y)_{\kappa}, u\right) \nu_{(x-y)_{\kappa}}(d u) \\
& +\frac{1}{2} \int\left(g(y+u)-g(y)-\langle\nabla g(y), u\rangle \mathbb{1}_{\{|u| \leq 1\}}\right) c\left(x, y,(y-x)_{\kappa}, u\right) \nu_{(y-x)_{\kappa}}(d u) \\
& +\int\left(g(y+z)-g(y)-\langle\nabla g(y), z\rangle \mathbb{1}_{\{|z| \leq 1\}}\right) \\
& \times\left(\tilde{\nu}_{x, y}-\frac{1}{2} \mu_{x, y,(x-y)_{\kappa}}-\frac{1}{2} \mu_{x, y,(y-x)_{\kappa}}\right)(d z) \\
& +\int\left(g(y+z)-g(y)-\langle\nabla g(y), z\rangle \mathbb{1}_{\{|z| \leq 1\}}\right) \tilde{c}(y, x, z) \nu(d z) \\
& =\frac{1}{2} \int\left(g(y+u)-g(y)-\langle\nabla g(y), u\rangle \mathbb{1}_{\{|u| \leq 1\}}\right) \mu_{x, y,(x-y)_{\kappa}}(d u) \\
& +\frac{1}{2} \int\left(g(y+u)-g(y)-\langle\nabla g(y), u\rangle \mathbb{1}_{\{|u| \leq 1\}}\right) \mu_{x, y,(y-x)_{\kappa}}(d u) \\
& +\int\left(g(y+z)-g(y)-\langle\nabla g(y), z\rangle \mathbb{1}_{\{|z| \leq 1\}}\right) \\
& \times\left(\tilde{\nu}_{x, y}-\frac{1}{2} \mu_{x, y,(x-y)_{\kappa}}-\frac{1}{2} \mu_{x, y,(y-x)_{\kappa}}\right)(d z) \\
& +\int\left(g(y+z)-g(y)-\langle\nabla g(y), z\rangle \mathbb{1}_{\{|z| \leq 1\}}\right) \tilde{c}(y, x, z) \nu(d z) \\
& =L g(y) \text {. }
\end{aligned}
$$

On the other hand, if $h(x, y)=f(x)$ for any $x, y \in \mathbb{R}^{d}$ and any $f \in C_{b}^{2}\left(\mathbb{R}^{d}\right)$, then we can easily see that $\widetilde{L} h(x, y)=L f(x)$. Combining with both conclusions above yields that the operator $\widetilde{L}$ defined by $(2.2)$ is a coupling operator of $L$.

The following lemma has been used in the proof above. 
Lemma 2.2. For any $z \in \mathbb{R}^{d}$ with $z \neq 0, \nu_{z}(d u)$ is a finite measure on $\left(\mathbb{R}^{d}, \mathscr{B}\left(\mathbb{R}^{d}\right)\right)$ such that

$$
\mu_{z}(d(u+z))=\mu_{-z}(d u)
$$

In particular,

$$
\mu_{z}\left(\mathbb{R}^{d}\right)=\mu_{-z}\left(\mathbb{R}^{d}\right) .
$$

Proof. The proof has been given in [22, Remark 2.1 and Corollary 6.2]. We omit it here.

2.2. Coupling process for Lévy type operators. The purpose of this part is to construct a coupling process associated with the coupling operator $\widetilde{L}$ given by $(2.2)$. Though the following argument is standard (see [21, Section 2] or [33, Section 2.2] for example), we still would like to present some details here.

Let $\mathscr{D}\left([0, \infty) ; \mathbb{R}^{d}\right)$ be the space of right continuous $\mathbb{R}^{d}$-valued functions having left limits on $[0, \infty)$ and equipped with the Skorokhod topology. For $t \geq 0$, denote by $X_{t}$ the projection coordinate map on $\mathscr{D}\left([0, \infty) ; \mathbb{R}^{d}\right)$. A probability measure $\mathbb{P}^{x}$ on the Skorokhod space $\mathscr{D}\left([0, \infty) ; \mathbb{R}^{d}\right)$ is said to be a solution to the martingale problem for $\left(L, C_{b}^{2}\left(\mathbb{R}^{d}\right)\right)$ with initial value $x \in \mathbb{R}^{d}$, if $\mathbb{P}^{x}\left(X_{0}=x\right)=1$ and, for every $f \in C_{b}^{2}\left(\mathbb{R}^{d}\right)$,

$$
\left\{f\left(X_{t}\right)-f(x)-\int_{0}^{t} L f\left(X_{s}\right) d s, t \geq 0\right\}
$$

is a $\mathbb{P}^{x}$-martingale. The martingale problem for $\left(L, C_{b}^{2}\left(\mathbb{R}^{d}\right)\right)$ is said to be wellposed if it has a unique solution for every initial value $x \in \mathbb{R}^{d}$. The definitions above are well adapted to the martingale problem for $\left(\widetilde{L}, C_{b}^{2}\left(\mathbb{R}^{2 d}\right)\right)$ with necessary modifications. We can refer to $[1,4,8,13,15,17,19,24,25,26,27,28,31]$ and the references therein for more details about martingale problem for non-local operators.

In order to prove the existence of the martingale problem for the coupling operator $\left(\widetilde{L}, C_{b}^{2}\left(\mathbb{R}^{2 d}\right)\right)$, we will write the coupling operator $\widetilde{L}$ into the form as the expression of Lévy type operator on $C_{b}^{2}\left(\mathbb{R}^{2 d}\right)$. For any $x, y \in \mathbb{R}^{d}$, and $A \in \mathscr{B}\left(\mathbb{R}^{2 d}\right)$, set

$$
\begin{aligned}
\widetilde{\nu}(x, y, A):= & \frac{1}{2} \int_{\left\{\left(z, z-(x-y)_{\kappa} \in A\right\}\right.} \mu_{x, y,(y-x)_{\kappa}}(d z)+\frac{1}{2} \int_{\left\{\left(z, z+(x-y)_{\kappa}\right) \in A\right\}} \mu_{x, y,(x-y)_{\kappa}}(d z) \\
& +\int_{\{(z, z) \in A\}}\left(\tilde{\nu}_{x, y}-\frac{1}{2} \mu_{x, y,(y-x)_{\kappa}}-\frac{1}{2} \mu_{\left.x, y,(x-y)_{\kappa}\right)}(d z)\right. \\
& +\int_{\{(z, 0) \in A\}} \tilde{c}(x, y, z) \nu(d z)+\int_{\{(0, z) \in A\}} \tilde{c}(y, x, z) \nu(d z) .
\end{aligned}
$$

Then, for any $x, y \in \mathbb{R}^{d}$ and $f \in C_{b}^{2}\left(\mathbb{R}^{2 d}\right)$,

$$
\begin{gathered}
\widetilde{L} f(x, y)=\int_{\mathbb{R}^{d} \times \mathbb{R}^{d}}\left(f\left((x, y)+\left(u_{1}, u_{2}\right)\right)-f(x, y)-\left\langle\nabla_{x} f(x, y), u_{1}\right\rangle \mathbb{1}_{\left\{\left|u_{1}\right| \leq 1\right\}}\right. \\
\left.-\left\langle\nabla_{y} f(x, y), u_{2}\right\rangle \mathbb{1}_{\left\{\left|u_{2}\right| \leq 1\right\}}\right) \widetilde{\nu}\left(x, y, d u_{1}, d u_{2}\right) .
\end{gathered}
$$

For any $h \in C_{b}\left(\mathbb{R}^{2 d}\right)$ and $x, y \in \mathbb{R}^{d}$, we have

$$
\begin{aligned}
& \int_{\mathbb{R}^{2 d}} h(u) \frac{|u|^{2}}{1+|u|^{2}} \widetilde{\nu}(x, y, d u) \\
& =\int_{\mathbb{R}^{d} \times \mathbb{R}^{d}} h\left(\left(u_{1}, u_{2}\right)\right) \frac{\left|u_{1}\right|^{2}+\left|u_{2}\right|^{2}}{1+\left|u_{1}\right|^{2}+\left|u_{2}\right|^{2}} \widetilde{\nu}\left(x, y, d u_{1}, d u_{2}\right)
\end{aligned}
$$




$$
\begin{aligned}
= & \frac{1}{2} \int_{\mathbb{R}^{d}} h\left(\left(z, z+(x-y)_{\kappa}\right)\right) \frac{|z|^{2}+\left|z+(x-y)_{\kappa}\right|^{2}}{1+|z|^{2}+\left|z+(x-y)_{\kappa}\right|^{2}} \mu_{x, y,(y-x)_{\kappa}}(d z) \\
& +\frac{1}{2} \int_{\mathbb{R}^{d}} h\left(\left(z, z-(x-y)_{\kappa}\right)\right) \frac{|z|^{2}+\left|z-(x-y)_{\kappa}\right|^{2}}{1+|z|^{2}+\left|z-(x-y)_{\kappa}\right|^{2}} \mu_{x, y,(x-y)_{\kappa}}(d z) \\
& +\int_{\mathbb{R}^{d}} h((z, z)) \frac{|z|^{2}+|z|^{2}}{1+|z|^{2}+|z|^{2}}\left(\tilde{\nu}_{x, y}-\frac{1}{2} \mu_{x, y,(x-y)_{\kappa}}-\frac{1}{2} \mu_{x, y,(y-x)_{\kappa}}\right)(d z) \\
& +\int_{\mathbb{R}^{d}} h((z, 0)) \frac{|z|^{2}}{1+|z|^{2}}(c(x, z)-c(x, z) \wedge c(y, z)) \nu(d z) \\
& +\int_{\mathbb{R}^{d}} h((0, z)) \frac{|z|^{2}}{1+|z|^{2}}(c(y, z)-c(x, z) \wedge c(y, z)) \nu(d z) .
\end{aligned}
$$

Since $c(x, z)$ is bounded and $(x, z) \mapsto c(x, z)$ is continuous, the function $(x, y) \mapsto$ $\int_{\mathbb{R}^{2 d}} h(u) \frac{|u|^{2}}{1+|u|^{2}} \widetilde{\nu}(x, y, d u)$ is continuous too. Therefore, by [31, Theorem 2.2], there is a solution to the martingale problem for $\left(\widetilde{L}, C_{b}^{2}\left(\mathbb{R}^{2 d}\right)\right)$, i.e., there are a probability space $\left(\widetilde{\Omega}, \widetilde{\mathscr{F}},\left(\widetilde{\mathscr{F}}_{t}\right)_{t \geq 0}, \widetilde{\mathbb{P}}\right)$ and an $\overline{\mathbb{R}}^{2 d}:=(\mathbb{R} \cup\{\infty\})^{2 d}$-valued process $\left(\widetilde{X}_{t}\right)_{t \geq 0}:=$ $\left(X_{t}^{\prime}, X_{t}^{\prime \prime}\right)_{t \geq 0}$ such that $\left(\widetilde{X}_{t}\right)_{t \geq 0}$ is $(\widetilde{\mathscr{F}})_{t \geq 0}$-progressively measurable, and for every $f \in C_{b}^{2}\left(\mathbb{R}^{2} d\right)$,

$$
\left\{f\left(\widetilde{X}_{t \wedge \widetilde{\zeta}}\right)-\int_{0}^{t \wedge \widetilde{\zeta}} \widetilde{L} f\left(\widetilde{X}_{s}\right) d s, t \geq 0\right\}
$$

is an $\left(\widetilde{\mathscr{F}_{t}}\right)_{t \geq 0}$-local martingale, where $\widetilde{\zeta}$ is the explosion time of $\left(\widetilde{X}_{t}\right)_{t \geq 0}$, i.e.,

$$
\widetilde{\zeta}=\lim _{n \rightarrow \infty} \inf \left\{t \geq 0:\left|X_{t}^{\prime}\right|+\left|X_{t}^{\prime \prime}\right| \geq n\right\} .
$$

By Proposition 2.1, $\widetilde{L}$ is the coupling operator of $L$, and so both distributions of the processes $\left(X_{t}^{\prime}\right)_{t \geq 0}$ and $\left(X_{t}^{\prime \prime}\right)_{t \geq 0}$ are solutions to the martingale problem of $L$. Since we assume that the martingale problem for $\left(L, C_{b}^{2}\left(\mathbb{R}^{d}\right)\right)$ is well-posed, the processes $\left(X_{t}^{\prime}\right)_{t \geq 0}$ and $\left(X_{t}^{\prime \prime}\right)_{t \geq 0}$ are non-explosive, and so we have $\widetilde{\zeta}=\infty$ a.e. That is, the coupling operator $\widetilde{\widetilde{L}}$ generates a non-explosive process $\left(\widetilde{X}_{t}\right)_{t \geq 0}$.

Let $T$ be the coupling time of $\left(X_{t}^{\prime}\right)_{t \geq 0}$ and $\left(X_{t}^{\prime \prime}\right)_{t \geq 0}$, i.e., $T=\inf \left\{t \geq 0: X_{t}^{\prime}=X_{t}^{\prime \prime}\right\}$. Then $T$ is an $(\widetilde{\mathscr{F}})_{t \geq 0}$-stopping time. Construct a new process $\left(Y_{t}^{\prime}\right)_{t \geq 0}$ as follows

$$
Y_{t}^{\prime}= \begin{cases}X_{t}^{\prime \prime}, & t<T \\ X_{t}^{\prime}, & t \geq T\end{cases}
$$

We can verify that $\left(Y_{t}^{\prime}\right)_{t \geq 0}$ is a solution to the martingale problem of $L$, see [33, Section 2.2]. Since the martingale problem for the operator $L$ is well posed, $\left(Y_{t}^{\prime}\right)_{t \geq 0}$ and $\left(X_{t}^{\prime \prime}\right)_{t \geq 0}$ are equal in the distribution. Therefore, we conclude that $\left(X_{t}^{\prime}, Y_{t}^{\prime}\right)_{t \geq 0}$ is also a non-explosive coupling process of $\left(X_{t}\right)_{t \geq 0}$ such that $X_{t}^{\prime}=Y_{t}^{\prime}$ for any $t \geq T$ and the generator of $\left(X_{t}^{\prime}, Y_{t}^{\prime}\right)_{t \geq 0}$ before the coupling time $T$ is just the coupling operator $\widetilde{L}$.

\section{COUpling APPROACH FOR REgularity OF SEMigroups}

3.1. Preliminary calculations. In the following, we assume that $\kappa \in(0,1]$. Let $\widetilde{L}$ be the coupling operator given above. We will estimate $\widetilde{L} f(|x-y|)$ for any $0 \leq f \in C_{b}([0, \infty)) \cap C^{2}((0, \infty))$ such that $f(0)=0$, and $f^{\prime} \geq 0$ and $f^{\prime \prime} \leq 0$ on $(0,2]$. 
For any $h \in C_{b}^{2}\left(\mathbb{R}^{2 d}\right)$, define

$$
\begin{aligned}
\widetilde{L}_{R} h(x, y)= & \int\left(h(x+z, y)-h(x, y)-\left\langle\nabla_{x} h(x, y), z\right\rangle \mathbb{1}_{\{|z| \leq 1\}}\right) \tilde{c}(x, y, z) \nu(d z) \\
& +\int\left(h(x, y+z)-h(x, y)-\left\langle\nabla_{y} h(x, y), z\right\rangle \mathbb{1}_{\{|z| \leq 1\}}\right) \tilde{c}(y, x, z) \nu(d z) .
\end{aligned}
$$

Set

$$
\widetilde{L}_{C}:=\widetilde{L}-\widetilde{L}_{R}
$$

Note that, if we define the following operator

$$
\begin{aligned}
L_{C} f(x) & :=\int\left(f(x+z)-f(x)-\langle\nabla f(x), z\rangle \mathbb{1}_{B(0,1)}(z)\right) \nu_{x, y}(d z) \\
& =\int\left(f(x+z)-f(x)-\langle\nabla f(x), z\rangle \mathbb{1}_{B(0,1)}(z)\right)(c(x, z) \wedge c(y, z)) \nu(d z),
\end{aligned}
$$

then, following the proof of Proposition 2.1, we can see that $\widetilde{L}_{C}$ is a coupling operator of $L_{C}$.

First, according to Lemma $2.2, \nu_{(y-x)_{\kappa}}(d z)$ is a finite measure on $\mathbb{R}^{d}$ for any $x, y \in \mathbb{R}^{d}$ with $x \neq y$. Then, for any $f \in C_{b}([0, \infty)) \cap C^{2}((0, \infty))$ and $x, y \in \mathbb{R}^{d}$ with $x \neq y$,

$$
\begin{aligned}
& \int\left(\left\langle\nabla_{x} f(|x-y|), z\right\rangle \mathbb{1}_{\{|z| \leq 1\}}\right. \\
& \left.+\left\langle\nabla_{y} f(|x-y|), z+(x-y)_{\kappa}\right\rangle \mathbb{1}_{\left\{\left|z+(x-y)_{\kappa}\right| \leq 1\right\}}\right) \mu_{x, y,(y-x)_{\kappa}}(d z) \\
& =\frac{f^{\prime}(|x-y|)}{|x-y|}\left(\int_{\{|z| \leq 1\}}\langle x-y, z\rangle \mu_{x, y,(y-x)_{\kappa}}(d z)\right. \\
& \left.-\int_{\left\{\left|z+(x-y)_{\kappa}\right| \leq 1\right\}}\left\langle x-y, z+(x-y)_{\kappa}\right\rangle \mu_{x, y,(y-x)_{\kappa}}(d z)\right) \\
& =\frac{f^{\prime}(|x-y|)}{|x-y|}\left(\int_{\{|z| \leq 1\}}\langle x-y, z\rangle \mu_{x, y,(y-x)_{\kappa}}(d z)\right. \\
& \left.-\int_{\{|z| \leq 1\}}\langle x-y, z\rangle \mu_{x, y,(x-y)_{\kappa}}(d z)\right),
\end{aligned}
$$

where in the last equality we used the fact that

$$
c\left(x, y,(y-x)_{\kappa}, u-(x-y)_{\kappa}\right)=c\left(x, y,(x-y)_{\kappa}, u\right), \quad x, y, u \in \mathbb{R}^{d}
$$

and Lemma 2.2. Similarly, it holds that

$$
\begin{aligned}
\int\left(\left\langle\nabla_{x} f(|x-y|), z\right\rangle \mathbb{1}_{\{|z| \leq 1\}}\right. & \\
& \left.+\left\langle\nabla_{y} f(|x-y|), z+(x-y)_{\kappa}\right\rangle \mathbb{1}_{\left\{\left|z+(x-y)_{\kappa}\right| \leq 1\right\}}\right) \mu_{x, y,(x-y)_{\kappa}}(d z) \\
=\frac{f^{\prime}(|x-y|)}{|x-y|}( & \int_{\{|z| \leq 1\}}\langle x-y, z\rangle \mu_{x, y,(x-y)_{\kappa}}(d z) \\
& \left.-\int_{\{|z| \leq 1\}}\langle x-y, z\rangle \mu_{x, y,(y-x)_{\kappa}}(d z)\right)
\end{aligned}
$$


Therefore, for any $x, y \in \mathbb{R}^{d}$ with $x \neq y$,

$$
\begin{aligned}
\widetilde{L}_{C} f(|x-y|)= & \frac{1}{2} \mu_{x, y,(x-y)_{\kappa}}\left(\mathbb{R}^{d}\right)(f(|x-y|+\kappa \wedge|x-y|)-f(|x-y|)) \\
& +\frac{1}{2} \mu_{x, y,(y-x)_{\kappa}}\left(\mathbb{R}^{d}\right)(f(|x-y|-\kappa \wedge|x-y|)-f(|x-y|)) \\
= & \frac{1}{2} \mu_{x, y,(x-y)_{\kappa}}\left(\mathbb{R}^{d}\right)[f(|x-y|+\kappa \wedge|x-y|) \\
& +f(|x-y|-\kappa \wedge|x-y|)-2 f(|x-y|)],
\end{aligned}
$$

where in the last equality we have used the fact that $\mu_{x, y,(x-y)_{\kappa}}\left(\mathbb{R}^{d}\right)=\mu_{x, y,(y-x)_{\kappa}}\left(\mathbb{R}^{d}\right)$.

Next, we assume that $f \geq 0$ with $f(0)=0$ on $[0, \infty)$, and $f^{\prime} \geq 0$ and $f^{\prime \prime} \leq 0$ on $(0,2]$. Let $\varepsilon_{0} \in(0, \kappa]$. Then, for any $\varepsilon \in\left(0, \varepsilon_{0}\right]$ and any $x, y \in \mathbb{R}^{d}$ with $|x-y| \leq \varepsilon$, we have

$$
\widetilde{L}_{C} f(|x-y|) \leq \frac{1}{2} J(|x-y|)(f(2|x-y|)-2 f(|x-y|)),
$$

where in the inequality above

$$
J(r):=\inf _{x, y \in \mathbb{R}^{d}:|x-y|=r} \mu_{x, y,(x-y)}\left(\mathbb{R}^{d}\right)
$$

and we have used the fact that

$$
f(2 r)=f(r)+\int_{r}^{2 r} f^{\prime}(s) d s=f(r)+\int_{0}^{r} f^{\prime}(s+r) d s \leq f(r)+\int_{0}^{r} f^{\prime}(s) d s=2 f(r)
$$

for any $r \in\left(0, \varepsilon_{0}\right]$.

We will give estimates for $\widetilde{L}_{R} f(|x-y|)$. For any $f \in C_{b}([0, \infty)) \cap C^{2}((0, \infty))$ with $f \geq 0, f^{\prime} \geq 0$ and $f^{\prime \prime} \leq 0$ on $(0,2]$. Then, for any $x, y \in \mathbb{R}^{d}$ with $x \neq y$,

$$
\begin{aligned}
& \widetilde{L}_{R} f(|x-y|) \\
& =\int\left(f(|x-y+z|)-f(|x-y|)-\frac{f^{\prime}(|x-y|)}{|x-y|}\langle x-y, z\rangle \mathbb{1}_{\{|z| \leq 1\}}\right) \\
& \quad \times(c(x, z)-c(x, z) \wedge c(y, z)) \nu(d z) \\
& +\int\left(f(|x-y-z|)-f(|x-y|)+\frac{f^{\prime}(|x-y|)}{|x-y|}\langle x-y, z\rangle \mathbb{1}_{\{|z| \leq 1\}}\right) \\
& \quad \quad \times(c(y, z)-c(x, z) \wedge c(y, z)) \nu(d z) \\
& =\int_{\{|z| \leq 1\}}\left(f(|x-y+z|)-f(|x-y|)-\frac{f^{\prime}(|x-y|)}{|x-y|}\langle x-y, z\rangle\right) \\
& \quad \times \quad(c(x, z)-c(x, z) \wedge c(y, z)) \nu(d z) \\
& \quad \int_{\{|z| \leq 1\}}\left(f(|x-y-z|)-f(|x-y|)+\frac{f^{\prime}(|x-y|)}{|x-y|}\langle x-y, z\rangle\right) \\
& \quad \times(c(y, z)-c(x, z) \wedge c(y, z)) \nu(d z) \\
& +\int_{\{|z|>1\}}(f(|x-y+z|)-f(|x-y|))(c(x, z)-c(x, z) \wedge c(y, z)) \nu(d z) \\
& +\int_{\{|z|>1\}}(f(|x-y-z|)-f(|x-y|))(c(y, z)-c(x, z) \wedge c(y, z)) \nu(d z) .
\end{aligned}
$$

We further consider the following two cases. 
(i) Since for any $a, b \in(0,2]$,

$$
f(b)-f(a) \leq f^{\prime}(a)(b-a),
$$

we have that for any $x, y, z \in \mathbb{R}^{d}$ with $0<|x-y| \leq \varepsilon_{0}$ and $|z| \leq 1$,

$$
\begin{aligned}
& f(|x-y+z|)-f(|x-y|)-\frac{f^{\prime}(|x-y|)}{|x-y|}\langle x-y, z\rangle \\
& \leq \frac{f^{\prime}(|x-y|)}{|x-y|}\left(|x-y+z||x-y|-|x-y|^{2}-\langle x-y, z\rangle\right) \\
& \leq \frac{f^{\prime}(|x-y|)}{|x-y|} \frac{|z|^{2}}{2},
\end{aligned}
$$

where the last inequality follows from the fact that

$$
\langle x-y, z\rangle=\frac{1}{2}\left(|x-y+z|^{2}-|x-y|^{2}-|z|^{2}\right), \quad x, y, z \in \mathbb{R}^{d} .
$$

This yields that for any $x, y \in \mathbb{R}^{d}$ with $0<|x-y| \leq \varepsilon_{0}$,

$$
\begin{aligned}
\widetilde{L}_{R} f(|x-y|) \leq & \left(\int_{\{|z| \leq 1\}}|c(x, z)-c(y, z)||z|^{2} \nu(d z)\right) \frac{f^{\prime}(|x-y|)}{|x-y|} \\
& +2\left(\int_{\{|z|>1\}} \nu(d z)\right)\left[\sup _{x, z \in \mathbb{R}^{d}:|z|>1} c(x, z)\right]\|f\|_{\infty} .
\end{aligned}
$$

(ii) If

$$
\int_{\{|z| \leq 1\}}|z| \nu(d z)<\infty,
$$

then for any $x, y, z \in \mathbb{R}^{d}$ with $0<|x-y| \leq \varepsilon_{0}$ and $|z| \leq 1$,

$$
f(|x-y+z|)-f(|x-y|)-\frac{f^{\prime}(|x-y|)}{|x-y|}\langle x-y, z\rangle \leq 2 f^{\prime}(|x-y|)|z| .
$$

Following the same argument as that in (i), we can arrive at for any $x, y \in \mathbb{R}^{d}$ with $0<|x-y| \leq \varepsilon_{0}$

$$
\begin{aligned}
\widetilde{L}_{R} f(|x-y|) \leq & 4\left(\int_{\{|z| \leq 1\}}|c(x, z)-c(y, z)||z| \nu(d z)\right) f^{\prime}(|x-y|) \\
& +2\left(\int_{\{|z|>1\}} \nu(d z)\right)\left[\sup _{x, z \in \mathbb{R}^{d:|z|>1}} c(x, z)\right]\|f\|_{\infty} .
\end{aligned}
$$

Combining all the estimates above, we can get that

Proposition 3.1. Let $0 \leq f \in C_{b}([0, \infty)) \cap C^{2}((0, \infty))$ such that $f(0)=0$, and $f^{\prime} \geq 0$ and $f^{\prime \prime} \leq 0$ on $(0,2]$. Let $0<\varepsilon_{0} \leq \kappa \leq 1$ and $J(r)$ be defined by (3.2). Then, for any $x, y \in \mathbb{R}^{d}$ with $0<|x-y| \leq \varepsilon_{0}$,

(1) it holds that

$$
\begin{aligned}
\widetilde{L} f(|x-y|) \leq & \frac{1}{2} J(|x-y|)(f(2|x-y|)-2 f(|x-y|)) \\
& +\left(\int_{\{|z| \leq 1\}}|c(x, z)-c(y, z)||z|^{2} \nu(d z)\right) \frac{f^{\prime}(|x-y|)}{|x-y|}
\end{aligned}
$$




$$
+2\left(\int_{\{|z|>1\}} \nu(d z)\right)\left[\sup _{x, z \in \mathbb{R}^{d:|z|>1}} c(x, z)\right]\|f\|_{\infty} .
$$

(2) if additionally (3.4) is satisfied, then

$$
\begin{aligned}
\widetilde{L} f(|x-y|) \leq & \frac{1}{2} J(|x-y|)(f(2|x-y|)-2 f(|x-y|)) \\
& +4\left(\int_{\{|z| \leq 1\}}|c(x, z)-c(y, z)||z| \nu(d z)\right) f^{\prime}(|x-y|) \\
& +2\left(\int_{\{|z|>1\}} \nu(d z)\right)\left[\sup _{x, z \in \mathbb{R}^{d:|z|>1}} c(x, z)\right]\|f\|_{\infty} .
\end{aligned}
$$

Remark 3.2. The estimates for $\widetilde{L} f(|x-y|)$ consist three terms. The first one comes from the operator $\widetilde{L}_{C}$, which is a leading part for our purpose. Other two terms are due to the operator $\widetilde{L}_{R}$.

3.2. General results. In the following, we present general results concerning the spatial regularity of semigroups.

Theorem 3.3. Assume that there is a nonnegative and $C_{b}([0, \infty)) \cap C^{3}((0, \infty))$ function $\psi$ such that

(i) $\psi(0)=0, \psi^{\prime} \geq 0, \psi^{\prime \prime} \leq 0$ and $\psi^{\prime \prime \prime} \geq 0$ on $(0,2]$;

(ii) For any constants $c_{1}, c_{2}>0$,

$$
\limsup _{r \rightarrow 0}\left[J_{\nu}(r) r^{2} \psi^{\prime \prime}(2 r)+c_{1} w(r) \psi^{\prime}(r) r^{-1}+c_{2}\right]<0,
$$

where

$$
J_{\nu}(r)=\inf _{z \in \mathbb{R}^{d}:|z|=r} \nu_{z}\left(\mathbb{R}^{d}\right)
$$

with $\nu_{z}(d z)$ defined in (2.1), and

$$
w(r)=\sup _{x, y \in \mathbb{R}^{d}:|x-y|=r}\left(\int_{\{|z| \leq 1\}}|c(x, z)-c(y, z)||z|^{2} \nu(d z)\right) .
$$

Then, there are constants $C, \varepsilon_{0}>0$ such that for all $f \in B_{b}\left(\mathbb{R}^{d}\right)$ and $t>0$,

$$
\sup _{x \neq y} \frac{\left|P_{t} f(x)-P_{t} f(y)\right|}{\psi(|x-y|)} \leq C\|f\|_{\infty} \inf _{\varepsilon \in\left(0, \varepsilon_{0}\right]}\left[\frac{1}{\psi(\varepsilon)}+\frac{1}{t \lambda_{\psi}(\varepsilon)}\right]
$$

where

$$
\lambda_{\psi}(\varepsilon):=-\sup _{0<r \leq \varepsilon} J_{\nu}(r) r^{2} \psi^{\prime \prime}(2 r) .
$$

Assume additionally that (3.4) holds. Then the conclusion above still holds, if (3.6) is replaced by

$$
\limsup _{r \rightarrow 0}\left[J_{\nu}(r) r^{2} \psi^{\prime \prime}(2 r)+c_{1} w_{*}(r) \psi^{\prime}(r)+c_{2}\right]<0
$$

where

$$
w_{*}(r)=\sup _{x, y \in \mathbb{R}^{d:|x-y|=r}}\left(\int_{\{|z| \leq 1\}}|c(x, z)-c(y, z)||z| \nu(d z)\right) .
$$


Proof. First, by (3.6), we have

$$
\limsup _{r \rightarrow 0} J_{\nu}(r) r^{2} \psi^{\prime \prime}(2 r)<0
$$

Due to $\psi^{\prime \prime \prime} \geq 0$ on $(0,2]$, it holds for any $0<r \leq 1$ that

$$
2 \psi(r)-\psi(2 r)=-\int_{0}^{r} \int_{s}^{r+s} \psi^{\prime \prime}(u) d u d s \geq-\psi^{\prime \prime}(2 r) r^{2} .
$$

Let $0<\varepsilon_{0}<\kappa \leq 1$ and $\varepsilon \in\left(0, \varepsilon_{0}\right]$. For any $x, y \in \mathbb{R}^{d}$ with $0<|x-y| \leq \varepsilon$, according to Proposition 3.1(1), we find that

$$
\widetilde{L} \psi(|x-y|) \leq \frac{1}{2} J(|x-y|) \psi^{\prime \prime}(2|x-y|)|x-y|^{2}+c_{1} w(|x-y|) \frac{\psi^{\prime}(|x-y|)}{|x-y|}+c_{2},
$$

where in the inequality above we used the facts that $c(x, z)$ is bounded from above and $\psi$ is bounded.

Since $c(x, z)$ is bounded from below, there is a constant $c_{3}>0$ such that for all $r>0, J(r) \geq c_{3} J_{\nu}(r)$. This further yields that for any $x, y \in \mathbb{R}^{d}$ with $0<|x-y| \leq \varepsilon$

$$
\begin{aligned}
\widetilde{L} \psi(|x-y|) & \leq \frac{c_{3}}{2} J_{\nu}(|x-y|) \psi^{\prime \prime}(2|x-y|)|x-y|^{2}+c_{1} w(|x-y|) \frac{\psi^{\prime}(|x-y|)}{|x-y|}+c_{2} \\
& \leq c_{4} J_{\nu}(|x-y|) \psi^{\prime \prime}(2|x-y|)|x-y|^{2} \\
& \leq-c_{4} \lambda_{\psi}(\varepsilon)
\end{aligned}
$$

where in the second inequality we used (3.6).

Having the inequality above at hand, we can obtain the first desired assertion by [20, Proposition 4.1]. The second desired assertion follows from the argument above and Proposition 3.1(2).

From Theorem 3.3, we can further deduce the time-space regularity of semigroups. In details, let $\left(X_{t}^{x}\right)_{t \geq 0}$ be the strong Markov process associated with the operator $L$ starting from $x$. Under assumptions of Theorem 3.3, for any $0<s<t, x, y \in \mathbb{R}^{d}$ and $f \in B_{b}\left(\mathbb{R}^{d}\right)$,

$$
\begin{aligned}
\left|P_{s} f(x)-P_{t} f(y)\right| & =\left|\mathbb{E}^{x} f\left(X_{s}\right)-\mathbb{E}^{y} f\left(X_{t}\right)\right|=\left|\mathbb{E}^{x} f\left(X_{s}\right)-\mathbb{E}^{y} \mathbb{E}^{X_{t-s}^{y}} f\left(X_{s}\right)\right| \\
& \leq \mathbb{E}^{y}\left|\mathbb{E}^{x} f\left(X_{s}\right)-\mathbb{E}^{X_{t-s}^{y}} f\left(X_{s}\right)\right| \\
& \leq C\|f\|_{\infty} \inf _{\varepsilon \in\left(0, \varepsilon_{0}\right]}\left[\frac{1}{\psi(\varepsilon)}+\frac{1}{t \lambda_{\psi}(\varepsilon)}\right] \mathbb{E}^{y} \psi\left(\left|x-X_{t-s}^{y}\right|\right),
\end{aligned}
$$

where in the second equality we used the Markov property, and the last inequality follows from (3.8). In order to estimate $\mathbb{E}^{y} \psi\left(\left|x-X_{t-s}^{y}\right|\right)$, one can refer to [16] for the recent study of moments estimates for Lévy-type processes. The details are omitted here.

3.3. Proofs. To prove Theorem 1.1, we also need the following lemma.

Lemma 3.4. Suppose that there are constants $\alpha \in(0,2)$ and $c_{0}>0$ such that

$$
\nu(d z) \geq \frac{c_{0}}{|z|^{d+\alpha}} \mathbb{1}_{V_{\xi}}(d z),
$$

where $V_{\xi}$ is defined by (1.4). Then, there are constants $c_{1}>0$ and $r_{0} \in(0,1)$ such that for all $0<r \leq r_{0}$,

$$
J_{\nu}(r) \geq c_{1} r^{-\alpha}
$$


where $J_{\nu}$ is defined by (3.7).

Proof. For any $z \in \mathbb{R}^{d}$, let $z=\left(z_{1}, z_{2}, \cdots, z_{d}\right)$. Without loss of generality, we may and can assume that $\xi=e_{1}=(1,0, \cdots, 0)$. Denote by

$$
q(z)=\frac{c_{0}}{|z|^{d+\alpha}} \mathbb{1}_{\left\{z_{1} \geq \delta|z|,|z| \leq 1\right\}}(z), \quad z \in \mathbb{R}^{d} .
$$

Then, for any $x, z \in \mathbb{R}^{d}$,

$$
q(z) \wedge q(x+z)=\mathbb{1}_{\left\{z_{1} \geq \delta|z|, z_{1}+x_{1} \geq \delta|x+z|,|z| \leq 1,|x+z| \leq 1\right\}}\left(\frac{c_{0}}{|z|^{d+\alpha}} \wedge \frac{c_{0}}{|z+x|^{d+\alpha}}\right) .
$$

In the following, we first suppose that $x_{1} \geq 0$. Hence, for any $x \in \mathbb{R}^{d}$ with $|x|$ small enough,

$$
\begin{aligned}
& \int q(z) \wedge q(x+z) d z \\
& \geq \int_{\left\{z_{1} \geq(1+\delta)|z| / 2,2 \delta|x| /(1-\delta) \leq|z| \leq \delta /(1+\delta)\right\}} \frac{c_{0}}{(|x|+|z|)^{d+\alpha}} d z \\
& \geq c_{1} \int_{\left\{z_{1} \geq(1+\delta)|z| / 2,2 \delta|x| /(1-\delta) \leq|z| \leq \delta /(1+\delta)\right\}} \frac{1}{|z|^{d+\alpha}} d z \\
& \geq c_{1} \int_{\left\{(1+\delta)\left(z_{2}^{2}+\cdots+z_{d}^{2}\right)^{1 / 2} /(1-\delta) \leq z_{1} \leq \delta /[2(1+\delta)], 2 \delta|x| /(1-\delta) \leq\left(z_{2}^{2}+\cdots+z_{d}^{2}\right)^{1 / 2} \leq \delta /[2(1+\delta)]\right\}} \frac{1}{|z|^{d+\alpha}} d z \\
& \geq c_{2} \int_{2 \delta|x| /(1-\delta)}^{c_{3}} d r \int_{(1+\delta) r /(1-\delta)}^{c_{4}} \frac{r^{d-2}}{\left(z_{1}+r\right)^{d+\alpha}} d z_{1} \\
& \geq c_{5}|x|^{-\alpha} .
\end{aligned}
$$

If $x_{1} \leq 0$, then, following the argument above, we have

$$
\begin{aligned}
& \int q(z) \wedge q(x+z) d z \\
& \geq \int_{\left\{z_{1} \geq(1+\delta)|z| / 2,2(1+\delta)|x| /(1-\delta) \leq|z| \leq(1+\delta) /(3+\delta)\right\}} \frac{c_{0}}{(|x|+|z|)^{d+\alpha}} d z \\
& \geq c_{6}|x|^{-\alpha} .
\end{aligned}
$$

Combining all the estimates above, we have obtained the desired assertion.

We are now in a position to give

Proof of Theorem 1.1. We will apply Theorem 3.3. For (1), noticing that $\alpha_{2} \in(1,2)$ too, we choose $\phi(r)=r\left(1-\log ^{-\theta}(1 / r)\right)$ for $r>0$ small enough, where $\theta$ is given in (1.5). For (2), we take $\phi(r)=r \log ^{\theta}(1 / r)$ for $r>0$ small enough, where $\theta>0$ if $\alpha_{1}>1$, and $\theta>1$ if $\theta_{1}=1$. For (3), since $\alpha_{2} \in(0,1),(3.4)$ holds. Then, we can take $\phi(r)=r^{\theta}$ for $r>0$ small enough. Therefore, with functions $\phi$ above, the desired assertion follows from Theorem 3.3.

Proof of Corollary 1.2. Let $u$ be a bounded harmonic function on $\mathbb{R}^{d}$. Then, for any $x, y \in \mathbb{R}^{d}$,

$$
u(x)-u(y)=P_{1} u(x)-P_{1} u(y) .
$$

This along with Theorem 1.1 immediately yields the desired assertion. 
Proof of Corollary 1.3. Since the proof is mainly based on that of [29, Theorem 2.1], we only point out necessary modifications here. For simplicity, we just consider the case (3) in Theorem 1.1. For any $\rho \geq 1$, let $v(x)=\rho^{-\beta} u(\rho x)$. Then, it is obvious that $P_{t} v(x)=v(x)$ for all $x \in \mathbb{R}^{d}$ and $t>0$; moreover, $\|v\|_{L^{\infty}\left(B_{R}(0), d x\right)} \leq c R^{\beta}$ with the same constant as $u$.

For any $M>0$, let $v_{M}(x)=v(x) \mathbb{1}_{\{|x| \leq M\}}$. Then, for any $x, y \in \mathbb{R}^{d}$, according to Theorem 1.1(3), we have

$$
\begin{aligned}
|v(x)-v(y)|= & \left|P_{1} v(x)-P_{1} v(y)\right| \\
\leq & \left|P_{1} v_{M}(x)-P_{1} v_{M}(y)\right|+\left|P_{1}\left(v-v_{M}\right)(x)\right|+\left|P_{1}\left(v-v_{M}\right)(y)\right| \\
\leq & c_{1}|x-y|^{\theta} M^{\beta} \\
& +\left|P_{1} v_{M}(x)-P_{1} v_{M}(y)\right|+\left|P_{1}\left(v-v_{M}\right)(x)\right|+\left|P_{1}\left(v-v_{M}\right)(y)\right| .
\end{aligned}
$$

On the other hand, under (1.3) (in particular, $\nu(d z) \leq \frac{c_{2}}{|z|^{d+\alpha_{2}}} d z$ ), for any $\varepsilon>0$ and the function $h(x)=\left(1+|x|^{2}\right)^{\left(\alpha_{2}-\varepsilon\right) / 2}$, we can check that there is a constant $c_{3}>0$ such that for all $x \in \mathbb{R}^{d}$,

$$
\operatorname{Lh}(x) \leq c_{3} h(x),
$$

which yields that $P_{1} h(x) \leq c_{4} h(x)$. Thus, for all $x \in B_{1}(0)$

$$
\begin{aligned}
\left|P_{1}\left(v-v_{M}\right)(x)\right| & \leq \int_{\{|z|>M\}}|v|(z) P_{1}(x, d z) \\
& \leq\left(\int_{\{|z|>M\}} h(z) P_{1}(x, d z)\right)\left(\sup _{z \in M} \frac{|v|(z)}{h(z)}\right) \\
& \leq c_{5} M^{-\left(\alpha_{2}-\beta-\varepsilon\right)} .
\end{aligned}
$$

In particular, taking $\varepsilon=\left(\alpha_{2}-\beta\right) / 2$, we arrive at that for all $x \in B_{1}(0)$,

$$
\left|P_{1}\left(v-v_{M}\right)(x)\right| \leq c_{5} M^{-\varepsilon} \text {. }
$$

Combining with all the estimates above, for any $x, y \in B_{1}(0)$,

$$
|v(x)-v(y)| \leq c_{6}\left(|x-y|^{\theta} M^{\beta}-M^{-\varepsilon}\right) .
$$

Letting $M=|x-y|^{-\theta /(\beta+\varepsilon)}$, we get that for any $x, y \in B_{1}(0)$,

$$
|v(x)-v(y)| \leq c_{7}|x-y|^{\gamma},
$$

where $\gamma=\varepsilon \theta /(\beta+\varepsilon)$. This shows the same conclusion as [29, (2.9)]. Furthermore, one can follow the argument of [29, Theorem 2.1] to prove the desired assertion.

Next, we present the

Proof of Theorem 1.4. Let $L$ be the operator given by (1.1), and let $L_{\mu}:=L_{*}-L$. Since $L_{*}$ is a linear operator, we can split the construction of coupling operator $L_{*}$ into those of $L$ and $L_{\mu}$. For $L$, we still use the coupling operator $\widetilde{L}$ defined by (2.2). For $L_{\mu}$, we define a coupling operator $\widetilde{L}_{\mu}$ as follows: for any $h \in C_{b}^{2}\left(\mathbb{R}^{2 d}\right)$,

$$
\begin{aligned}
\widetilde{L}_{\mu} h(x, y)= & \int\left(h(x+z, y+z)-h(x, y)-\left\langle\nabla_{x} h(x, y), z\right\rangle \mathbb{1}_{\{|z| \leq 1\}}\right. \\
& \left.-\left\langle\nabla_{y} h(x, y), z\right\rangle \mathbb{1}_{\{|z| \leq 1\}}\right) \mu(x, d z) \wedge \mu(y, d z) \\
+ & \left(h(x+z, y)-h(x, y)-\left\langle\nabla_{x} h(x, y), z\right\rangle \mathbb{1}_{\{|z| \leq 1\}}\right)
\end{aligned}
$$




$$
\begin{gathered}
\times(\mu(x, d z)-\mu(x, d z) \wedge \mu(y, d z)) \\
+\int\left(h(x, y+z)-h(x, y)-\left\langle\nabla_{y} h(x, y), z\right\rangle \mathbb{1}_{\{|z| \leq 1\}}\right) \\
\times(\mu(y, d z)-\mu(x, d z) \wedge \mu(y, d z)) .
\end{gathered}
$$

Then, we can follow the proof of Proposition 2.1 and obtain that $\widetilde{L}_{*}:=\widetilde{L}+\widetilde{L}_{\mu}$ is a coupling operator of $L$. Furthermore, using the assumption that for any $h \in C_{b}^{2}\left(\mathbb{R}^{d}\right)$, the function $x \mapsto \int_{\mathbb{R}^{d}} h(z) \frac{|z|^{2}}{1+|z|^{2}} \mu(x, d z)$ is continuous, and repeating the argument in Section 2.2, we can prove the existence of coupling process associated with the coupling operator $\widetilde{L}_{*}$ above.

Next, for any $0 \leq f \in C_{b}([0, \infty)) \cap C^{2}((0, \infty))$ such that $f(0)=0$, and $f^{\prime} \geq 0$ and $f^{\prime \prime} \leq 0$ on $(0,2]$, we will adopt the arguments of (3.3) and (3.5) to obtain some similar estimates about $\widetilde{L}_{\mu} f(|x-y|)$. With these at hand and estimates for $\widetilde{L}$ in Proposition 3.1, one can obtain the desired conclusion by using Theorem 3.3 and following the proof of Theorem 1.1 line by line.

Acknowledgements. The research is supported by National Natural Science Foundation of China (No. 11522106), Fok Ying Tung Education Foundation (No. 151002), and the Program for Nonlinear Analysis and Its Applications (No. IRTL1206).

\section{REFERENCES}

[1] Abels, H. and Kassmann, M.: The Cauchy problem and the martingale problem for integrodifferential operators with non-smooth kernels, Osaka J. Math. 46 (2009), 661-683.

[2] Barles, G., Chasseigne, E., Ciomaga, A. and Imbert, C.: Lipschitz regularity of solutions for mixed integro-differential equations, J. Differential Equations 252 (2012), 6012-6060.

[3] Barles, G., Chasseigne, E. and Imbert, C.: Hölder continuity of solutions of second-order non-linear elliptic integro-differential equations, J. Eur. Math. Soc. 13 (2011), 1-26.

[4] Bass, R.F.: Uniqueness in law for pure jump type Markov processes, Probab. Theory Relat. Fields 79 (1988), 271-287.

[5] Bass, R.F. and Kassmann, M.: Harnack inequalities for non-local operators of variable order, Trans. Amer. Math. Soc. 357 (2005), 837-850.

[6] Bass, R.F. and Kassmann, M.: Hölder continuity of harmonic functions with respect to operators of variable order, Comm. PDE 30 (2005), 1249-1259.

[7] Bass, R.F. and Levin, D.A.: Harnack inequality for jump processes, Potential Anal. 17 (2002), 375-388.

[8] Bass, R.F. and Tang, H.L.: The martingale problem for a class of stable-like processes, Stochastic Process. Appl. 119 (2009), 1144-1167.

[9] Böttcher, B., Schilling, R.L. and Wang, J.: Lévy-Type Processes: Construction, Approximation and Sample Path Properties, Lecture Notes in Mathematics, vol. 2099, Lévy Matters III, Springer, Berlin 2014.

[10] Budhiraja, A., Chen, J. and Dupuis, P.: Large deviations for stochastic partial differential equations driven by a Poisson random measure, Stoch. Proc. Appl. 123 (2013), 523-560.

[11] Caffarelli, L. and Silvestre, L.: Regularity theory for fully nonlinear integro-differential equations, Comm. Pure Appl. Math. 62 (2009), 597-638.

[12] Caffarelli, L. and Silvestre, L.: The Evans-Krylov theorem for nonlocal fully nonlinear equations, Ann. of Math. 174 (2011), 1163-1187.

[13] Chen, Z.-Q. and Zhang, X.: Uniqueness of stable-like processes, arXiv:1604.02681.

[14] Kassmann, M., Rang, M. and Schwab, R.W.: Integro-differential equations with nonlinear directional dependence, Indiana Univ. Math. Journal 63 (2014), 1467-1498.

[15] Komatsu, T.: On the martingale problem for generators of stable processes with perturbations, Osaka J. Math. 21 (1984), 113-132. 
[16] Kühn, F.: Existence and estimates of moments for Lévy-type processes, Stoch. Proc. Appl. 127 (2017), 1018-1041.

[17] Kurtz, T.G.: Equivalence of stochastic equations and martingale problems, in: Crisan, D. (ed.): Stochastic Analysis in 2010, pp. 113-130, Springer, London, 2011.

[18] Kurtz, T.G. and Protter, P.E.: Weak convergence of stochastic integrals and differential equations. II. Infinite-dimensional case, (Montecatini Terme, 1995), Lecture Notes in Math., vol. 1627, pp. 197-285, Springer, Berlin, 1996.

[19] Lepeltier, J.-P. and Marchal, B.: Problème des martingales et équations différentielles stochastiques associées à un opérateur intégro-différential, Ann. Inst. Henri Poincaré 12 (1976), 43-103.

[20] Liang, M. and Wang, J.: Gradient estimates and ergodicity for SDEs driven multiplcative Lévy noises via coupling, arXiv:1801.05936

[21] Luo, D. and Wang, J.: Coupling by reflection and Hölder regularity for non-Local operators of variable order, to appear in Trans. Amer. Math. Soc.

[22] Luo, D. and Wang, J.: Refined basic couplings and Wasserstein-type distances for SDEs with Lévy noises, arXiv:1604.07206

[23] Jacob, N.: Pseudo-differential Operators and Markov Processes, Vol. 1 - Vol. 3, Imperial College Press, London 2001 - 2005.

[24] Jin, P.: Well-posedness of the martingale problem for non-local perturbations of Lévy-type generators, arXiv:1708.04467

[25] Jin, P.: Uniqueness in law for stable-like processes of variable order, arXiv:1802.01151

[26] Mikulevicius, R. and Pragarauskas, H.: On the martingale problem associated with nondegenerate Lévy operators, Lith. Math. J. 32 (1992), 297-311.

[27] Mikulevicius, R. and Pragarauskas, H.: On the Cauchy problem for integro-differential operators in Hölder classes and the uniqueness of the martingale problem, Potential Anal. 40 (2014), 539-563.

[28] Mikulevcius, R. and Pragarauskas, H.: On the Cauchy problem for integro-differential operators in Sobolev classes and the martingale problem, J. Diff. Eq. 256 (2014), 1581-1626.

[29] Ros-Oton, X. and Serra, J.: Regularity theory for general stable operators, J. Diff. Eq. 260 (2016), 8675-8715.

[30] Situ, R.: Theory of Stochastic Differential Equations with Jumps and Applications: Mathematical and Analystical Techniques with Applications to Eigineering, Springer, New York 2005.

[31] Stroock, D.: Diffusion processes associated with Lévy generators, Z. Wahrscheinlichkeitstheorie verw. Gebiete 32 (1975), 209-244.

[32] Wang, J.: On the existence and explicit estimates for the coupling property of Lévy processes with drift, J. Theor. Probab. 27 (2014), 1021-1044.

[33] Wang, J.: $L^{p}$-Wasserstein distance for stochastic differential equations driven by Lévy processes, Bernoulli 22 (2016), 1598-1616.

[34] Xie, L.: Singular SDEs with critical non-local and non-symmetric Lévy type generator, Stoch. Proc. Appl. 127 (2017), 3792-3824.

[35] Xie, L. and Xu, L.: Irregular stochastic differential equations driven by a family of Markov processes, arXiv:1610.07248 